\title{
Philosophy of Chemistry-A New Interdisciplinary Field?
}

\author{
Eric R. Scerri \\ Department of Chemistry, Purdue University, W est Lafayette, IN 47907; scerri@purdue.edu
}

W hat could possibly be the connection between chemistry and philosophy, apart from the obvious superficial one of their both representing quests for knowledge? $\mathrm{H}$ ow do contemporary chemists and philosophers generally view one another? These are some of the questions I will try to put before going on to describe the connections that have recently been forged between these two seemingly very diverse fields of academic study.

\section{The View from Each Side}

I think it is fair to say that chemists and philosophers traditionally regard one another with a certain amount of suspicion and in some cases maybe even a little contempt. Chemists rightly feel proud of the fact that they engage the phenomenal world through experimentation and are prepared to revise their theories and practices accordingly. To the chemist, the philosopher- who conducts no experiments whatsoever - is not worthy of very high esteem. From the scientific perspective, philosophical views do not seem very dynamic, since they sometimes stem from established philosophical doctrines or a priori beliefs about the ways the world should be.

Philosophers for their part are proud of their training in rigorous ways of thinking. They freely admit to not engaging in the grubby details of the experimental world because such activities might limit the generalities of their claims and their attempts to depict real ity in its broadest terms. Some of them may even secretly look down at chemists for taking scientific models literally, for being naive realists, and perhaps - to use some well-known pejorativelabels - for being "stamp collectors" and "pot boilers". Of course all these introductory remarks are intended by way of caricature; but although it is simplistic to accuse educated folk of such narrowmindedness, I believe there is at least some truth in what I am suggesting.

M ost philosophers of science believe that chemistry has been reduced to physics and is therefore of no fundamental interest. They believe that chemistry has no "big ideas" to compare with quantum mechanics and relativity in physics and $D$ arwin's theory in biology. Furthermore, given their relative lack of interest in experiment, as opposed to theory, it is not surprising that philosophers have tended to ignore that very experimental science-chemistry.

\section{New Thoughts on Chemistry}

It may therefore come as something of a surprise to the reader to learn that during the past ten years or so there has been a veritable upsurge of interest in the field of philosophy of chemistry. All the above misconceptions have begun to be questioned and philosophers of science now realize that while they have paid great attention to physics and more recently biology, they have almost completely neglected the central science of chemistry (1).

At the same time, several chemists and commentators have produced deeply reflective books and articles in which they explore the essential nature of chemistry and the chemist's practices and particular way of thinking (2). There are now both an international society and two international journals $s^{1}$ devoted to philosophical aspects of chemistry. In addition, a lively Internet discussion list called "philchem" has been in existence for about three years. ${ }^{2}$

\section{The Q uestion of "Reduction" in Chemistry}

Among the works produced by philosophers of chemistry is a critical analysis of the question of the reduction ${ }^{3}$ of chemistry to physics (3), something that seemed to have been regarded as a foregone conclusion by the previous generation of philosophers (4). The term reduction refers to the increasingly prevalent view, which has had a large impact on chemical education, that all the deep questions in science can be resolved by appealing to the more fundamental theories found in physics. The notion that chemistry does indeed reduce to physics is implicit in the increasing use of physical principles to explain atomic structure and the periodic system, for example. Similarly, many other areas of chemistry, including inorganic chemistry, are approached through physical principles rather than by focusing on qualitative aspects and the diversity of observed phenomena. As educators at all levels are well aware, part of the art of teaching chemistry lies in knowing how to maintain a balance between these unifying principles and the more apparently diverse qualitative and descriptive aspects. This issue is al so important to writers of textbooks and those designing instructional educational software.

O ne aspect of recent work in philosophy of chemistry is a willingness to explore the alleged reduction of chemistry to physics by examining just how much can be derived from ab initio quantum chemistry instead of the more traditional philosophical approach, which involves the axiomatization of theories and laws. The archetypal example of an axiomatic system is Euclidean geometry, in which the entire system can be represented as a set of precise and clearly stated rules. Any competent user of these rules can prove other propositions within the system with the utmost clarity and certainty. Similar axiomatic approaches developed in physics may have some value in establishing whether thermodynamics is reduced to statistical mechanics, for example, since some reasonably satisfactory formalized versions of these two branches of physics are available. H owever, in the case of chemistry, at least two problems render this approach to the question of reduction highly problematic. First, it is by no means clear that chemistry possesses any exact laws of the same form as $\mathrm{N}$ ewton's laws of motion or other physical laws. Second, the notion of axiomatizing chemistry appears to be rather ill founded, given the relative lack of rigorous mathematical 
theories in the chemical sciences (5). This is why philosophers of chemistry have adopted thealternative approach mentioned above and have examined the extent to which chemical data can be predicted from first principles. $O$ ne of them suggests that some quantum chemists overstate their claims to having reduced chemistry to quantum mechanics, even in the limited domain of calculating energies or bond angles, and that chemical educators need to take note of this situation (6).

\section{But Chemistry Does Have Laws}

I am not trying to imply that chemistry does not possess any laws. However, the periodic law of the chemical elements, for example, differs from typical laws in physics in that the recurrence of elements after certain interval s is only approximate. In addition, the repeat period varies as one progresses through the periodic system. These features do not render the periodic law any less lawlike, but they do suggest that the nature of laws may differ from one area of science to another. Perhaps chemical laws should not be judged to be deficient by the standards of laws of physics.

The periodic law and the associated periodic system represent what I believe to be one of chemistry's "big ideas". I do not need to stress to this readership the ubiquity of the periodic system in teaching and chemical research, and the historical role it has played in the foundation of atomic physics and quantum mechanics through the contributions of Thomson, Bohr, Pauli, and others (7).

\section{Models and Explanations in Chemistry}

Similarly, the nature of chemical models is providing a rich source of examples to philosophers who are interested in obtaining a wider view of these scientific entities. In addition, chemical educators have been drawn into philosophy of chemistry in an attempt to clarify the meaning of terms like model and law as employed in teaching chemistry (8). This kind of research is clearly needed in chemistry, which, to the outsider, appears to be guilty at times of adopting conflicting models to explain particular chemical facts. H ow many of us have experienced students' frustration when we give different chemical explanations depending on the context in which one and the same phenomenon is being discussed?

If one believes only in fundamental explanations, this form of activity appears to be seriously mistaken. H owever, as chemists we are al so aware of the need to operate on many levels and the fact that explanations can be genuinely levelspecific. Such approaches must be used very carefully. They should not degenerate into the introduction of ad hoc explanations that are invoked in the explanation of particular chemical facts but cannot be generalized to other situations. O ne example is the wide variety of explanations given for the apparent orbital paradox concerning the relative occupation and ionization of the $4 \mathrm{~s}$ and $3 \mathrm{~d}$ levels in the first transition metal series. The paradox I allude to is that the $4 \mathrm{~s}$ orbital is preferentially occupied but also preferentially ionized. $\mathrm{N}$ obody has yet rationalized this situation at a level that might be appropriate for teaching general chemistry. M ost educators and textbooks continue to argue that the $4 \mathrm{~s}$ orbital is preferentially occupied because it has a lower energy than $3 d$, in spite of several articles published in this Journal that state that the $4 \mathrm{~s}$ orbital never has a lower energy than the $3 d(9)$.

Another response, encountered particularly among theo- reticians, is that this is a futile question because the concept of orbitals ceases to refer to any objective entities in more advanced calculations and can only be maintained at the level of the $\mathrm{H}$ artree-Fock approximation. I suggest that this kind of response is just another way of expressing D irac's famous dictum whereby chemistry has been explained in principle by quantum mechanics. Such a response amounts to evading the issue, which is to try to obtain a consistent explanation within an orbital approximation such as the $\mathrm{H}$ artree-Fock model, since within this regime the concept of an orbital is well defined.

\section{Realism}

\section{Realism and Molecular Structure}

The question of realism regarding scientific terms in chemistry has al so been revisited in recent articles in philosophy of chemistry. For example, the commitment to realism, which some believe to be a feature of chemistry, has received a serious challenge in the form of the molecular structure controversy that has been the feature of at least two articles in this Journal (10).

W oolley and other authors suggest that the concept of molecular structure, which is so central to modern chemistry, is nothing but a metaphor having no objective reality at the quantum mechanical level. The basis of this claim lies in the fact that the appropriate $\mathrm{H}$ amiltonian used in quantum mechanical calculations for a molecule such as $\mathrm{C}_{3} \mathrm{H}_{4}$ only contains terms describing interactions between protons and electrons in the system. Woolley claims that the structure of the molecule (or the relative positions of the nuclei) is introduced somewhat artificially in calculation s by invoking the Born-O ppenheimer approximation, which assumes that only the electrons move within a rigid framework defined by the positions of thenuclei, which areassumed to be fixed in space. This approximation is based on the large differences in mass between electrons and nuclei, with the assumption that the electrons can respond instantaneously to changes in position of the nuclei.

Woolley and others have claimed that a purely quantum mechanical description involving the raw molecular $\mathrm{H}$ amiltonian without use of the Born-O ppenheimer approximation does not require the attribution of any structure to molecules. The sharing of the same $\mathrm{H}$ amiltonian by the three known isomers of $\mathrm{C}_{3} \mathrm{H}_{4}$ (see structures below) implies that a purely quantum mechanical calculation without the Born-O ppenheimer approximation cannot distinguish between these structures. $\mathrm{N}$ or, it is claimed, are such considerations purely academic; as Woolley pointed out, in many cases calculations carried out without the Born- 0 ppenheimer approximation yield more accurate predictions than those carried out by the more conventional approach, which doesmakeuse of the approximation.

$$
\begin{array}{ccc}
\mathrm{H}_{3} \mathrm{C}-\mathrm{C} \equiv \mathrm{CH}_{2} \quad \mathrm{H}_{2} \mathrm{C}=\mathrm{C}=\mathrm{CH}_{2} & \text { allene } \\
\text { methyl acetylene } & \text { cyclopropene }
\end{array}
$$

M ost chemists react with complete incredulity to the view that structure is nothing but a metaphor, pointing out the seemingly overwhelming evidence for structure that comes 
from spectroscopic and other structural studies. They suggest that if a deep quantum mechanical analysis reveals molecular structure to be a mathematical artifact, then the fault must lie with present-day quantum mechanics and not with the deeply entrenched chemical notion of structure. Interestingly, a philosopher of science has sided with the traditional chemical view in upholding the reality of molecular structure. Ramsey argued that careful analysis of the work of Woolley and some other authors reveals that they are themselves misinterpreting what it means to hold realistic views about scientific entities, but this debate is far from being completed (11).

\section{W hen Is Realism A ppropriate?}

I believe that chemists do in fact have a tendency to be naive realists and that while this philosophical attitude is perfectly appropriate in some instances it is not in others. It is appropriate in the sense that chemists operate with what have been termed secondary properties such as colors and smells. For example, the color of chlorine is correctly regarded in chemistry as simply "green", without specifying the exact frequency of its color. Properties such as color can be usefully regarded as characteristic of the compounds themselves in much chemical work. H owever, when dealing with properties that originate in quantum mechanics, such as atomic orbitals, should chemists defer to the physicist and accept what quantum mechanics might have to say on the subject?T his is what I formerly recommended, but I no longer believe that matters are so simple (12).

In modern chemistry such terms as caloric and phlogiston have long been dismissed because they are thought to be nonreferring; that is, we believe that nothing exists in the world to correspond to such terms, which were formerly invoked in chemical explanations. N evertheless, the term atomic orbitalwhich is also strictly nonreferring, unless one is concerned with the hydrogen atom - continues to be used in chemistry. In fact, orbitals and the related concept of electronic configurations, neither of which truly "exist" in many-electron atoms according to a strict interpretation of quantum me chanics, have become the central paradigm at all levels of chemistry.

\section{Can O rbitals Be Real in Chemistry but N ot in Physics?}

This situation raises a philosophical question regarding the status of orbitals and configurations. Although they may not exist in the context of quantum mechanics, both concepts serve as a very useful approximation, which clearly should not be abandoned. This issue is complicated by the fact that computational chemists use orbitals and configurations as mathematical fictions, whereas chemical educators tend to attribute something of a definite existence to them. Perhaps the current emphasis on teaching chemistry in a quasi-deductive manner, starting from the configurations of the elements, does chemistry a disservice by making an approximate model appear to be more concrete than it deserves. This situation is exacerbated by the tendency of some chemistry instructors and textbook authors to teach electronic configurations by means of drill exercises, thus further incorrectly suggesting the fundamental nature of orbitals and configurations. O ne rather extreme example of this tendency for drilling configurations into students can be seen in the otherwise excellent World of Chemistry video series.

If one takes a fundamentalist approach, it emerges that quantum mechanics denies the very existence of orbitals and configurations in many-electron atoms, rather than underwriting them as is generally supposed. H owever, chemistry is not physics, and it may well be that an approximate concept is very useful in chemistry despite being deemed nonexistent by the more fundamental discipline of physics. The way out of this impasse might be to uphold the autonomy of chemistry and to use what might be termed "chemists' orbitals". These entities would correspond to the electron orbits discussed just prior to the discovery of quantum mechanics, when orbits could still be regarded as "real" entities. In the case of these chemists' orbits one would need to retain most of the results from quantum mechanics, such as the probabilistic interpretation, but one might want to ignore the modern quantum mechanical finding that the assignment of four quantum numbers to each electron is strictly invalid.

Such a view of autonomous levels of science, which is gaining prominence in philosophy of science, would imply that chemistry can make use of fundamental physics by borrowing such terms as orbits and configurations but would not be required to follow strict physical laws to the point of denying the existence of atomic orbitals in chemistry (13).

\section{W here Is Naive Realism Inappropriate?}

Fritz Paneth, one of the founders of radiochemistry and a highly reflective scientist, once suggested that although chemists have much to gain from adopting a naively realistic attitude in refusing to follow the latest discoveries from physics at every turn, there is also a price to be paid. According to Paneth, chemists must abandon naive realism if they are to make any sense of the notion that elements persist in compounds they might form. For example, sodium as the gray metallic substance is nowhere to be found in the compound sodium chloride. Paneth's suggestion for overcoming this problem is to argue for a metaphysical or transcendental view of the elements as abstract bearers of properties that are themselves unobservable. As Paneth reminds us, it was this understanding of the term element, sometimes referred to as "basic substance" rather than the directly observable or "simple substance", that M endeleev adhered to when he constructed his periodic system.

Paneth also suggested that it was because $M$ endeleev held this kind of metaphysical view of the elements that he believed so strongly in the periodic law, even in instances where experimental evidence appeared to contradict it (14). Perhaps it is not that M endeleev's contemporaries just lacked the courage to make predictions, as many historians have claimed, but that they did not possess the appropriate philosophical attitude regarding the nature of the elements.

These days any high school student is likely to open a textbook and find ST M images of atoms and molecules. $\mathrm{He}$ or she might then be perplexed by hearing the debate surrounding the real ity or otherwise of atoms that has taken place over the years. H owever, as chemistry becomes increasingly sophisticated the need for philosophical analysis of all aspects of the subject, including perhaps the true significance of ST M images, will continue to increase. As someone once said, philosophy begins with the recognition of the difference between appearance and reality. ${ }^{4}$ 


\section{Black Boxes in Chemical Education}

Finally, the direction taken by contemporary research in chemical education has been cause for concern for some of us. Instead of focusing on the content of chemistry courses, the new tendency is to reach for cognitive psychology and allied fields and to concentrate almost exclusively on the "learning process" (15). The message appears to be that we mistakenly regard the mind of the student as a form of black box into which new information can be transferred intact by the teacher. The new approach is to explore this cognitive black box and to regard it as playing the central role in chemical education. But the enthusiasm with which this program has been embraced has meant that we are rapidly approaching the point where the content of chemistry courses is regarded as a new black box, which is therefore ignored while all attention is focused on how students learn.

I hope that a new interest in philosophy of chemistry may provide the impetus required in chemical education to redress the balance of focus back toward chemistry itself.

\section{Notes}

1. These journals are called Foundati ons of Chemistry and $\mathrm{H}$ yle. The author is editor-in-chief of Foundations of Chemistry, which invites submissions on philosophical, historical, educational, cultural, and conceptual aspects of chemistry. See Web page at http://www.wkap.nl/ journals/foch.

2. To subscribe to philchem send email message to listserv@ vm.sc.edu and write just the following in your subscription message: subscribe philchem your name.

3. There is no implied connection with reduction in the sense of the opposite of oxidation!

4. This issue has recently surfaced again, with the claim that atomic orbitals have been directly observed.

\section{Literature Cited}

1. Synthese 1997, 111, 211-324 and other papers in this special issue on philosophy of chemistry.

2. H offmann, R. The Same and N ot the Same; Columbia University Press: N ew York, 1995. Knight, D. Ideas in Chemistry, Rutgers University Press: N ew Brunswick, N J, 1992. N ye, M. J. From Chemical Philosophy to Theoretical Chemistry; University of California Press: Berkeley, 1993. Laszlo, P. La Parole des Choses; Collection Savoir-Science: Paris, 1993. H offmann, R.; Laszlo, P. Angew. Chem., Int. Ed. Engl. 1991, 30, 1-16.

3. Scerri, E. R.; M cl ntyre, L. Synthese 1997, 111, 213-232.

4. Kemeney, J. G.; O ppenheim, P. Phil. Stud. 1956, 7, 6-19.

5. Scerri, E. R. Erkenntnis 1997, 47, 229-243.

6. Scerri, E. R. Int. Stud. Philos. Sci. 1998, 12, 33-44.

7. Scerri, E. R. Am. Sci. 1997, 85, 546-553. Scerri, E. R. Sci. Am. 1998, 279(Sep), 78-83.

8. Bhushan, N .; Rosenfeld, S. J. Chem. Educ. 1995, 72, 578-582.

9. Melrose, M. P.; Scerri, E. R. J. Chem. Educ. 1996, 73, 498-503. Vanquickenbourne, L. G.; Pierloot, K.; D evoghel, D. J. Chem. Educ. 1994, 71, 469-471.

10. W eininger, S. J. J. Chem. Educ. 1984, 61, 939-944. Woolley, R. G. J. Am. Chem. Soc. 1978, 100, 1073-1078.

11. Ramsey, J. L. Synthese 1997, 111, 233-251.

12. Scerri, E. R. Br. J. Philos. Sci. 1991, 42, 309-325.

13. Scerri, E. R. Sci. \& Educ. 2000, 9(5), in press.

14. Paneth, F. A. Br. J. Philos. Sci. 1962, 13, 1-14, 144-160.

15. Spencer, J. N. J. Chem. Educ. 1999, 76, 566-569. G abel, D. J. Chem. Educ. 1999, 76, 548-554. 